\title{
A heat and mass transfer problem for the dissolution of an alumina particle in a cryolite bath
}

\author{
A. Kovács ${ }^{\mathrm{a}, *}$, C.J.W. Breward ${ }^{\mathrm{a}}$, K.E. Einarsrud ${ }^{\mathrm{c}}$, S.A. Halvorsen ${ }^{\mathrm{b}}$, E. Nordgård-Hansen ${ }^{\mathrm{b}}$, E. Manger $^{\mathrm{d}}$, A. Münch ${ }^{\mathrm{a}}$, \\ J.M. Oliver ${ }^{\mathrm{a}}$ \\ ${ }^{a}$ Mathematical Institute, University of Oxford, United Kingdom \\ ${ }^{b}$ Department of Technology, NORCE Norwegian Research Centre, Norway \\ ${ }^{c}$ Department of Materials Science and Engineering, Norwegian University of Science and Technology (NTNU), Norway \\ ${ }^{d}$ Hydro Aluminium, Norway
}

\begin{abstract}
We investigate the spherically symmetric dissolution of an initially cold alumina particle in a bath of molten cryolite. The cryolite initially freezes on the particle, forming a shell that must melt before the particle can dissolve. We derive asymptotic solutions valid in the limits of small-superheat and of small Stefan number. In the small-superheat limit, the evolution of the boundary exhibits a two-scale behaviour. In the small Stefan number limit, we find that the behaviour of a particle could be limited by either the dissolution (in the case where the temperature differences are small) or by heat transfer (when the latent heat is large and the temperature gradients are large). Our asymptotic predictions are validated by a front fixing numerical scheme that we initiate using the early-time asymptotics.
\end{abstract}

Keywords: alumina feeding, asymptotic analysis, mathematical modelling, moving boundary, Stefan-problem

\section{Introduction}

Since its introduction in 1886, the Hall-Héroult process has made aluminium metal into a commodity product. In this continuous process, alumina is dissolved in a cryolite bath from which aluminium is produced by electrolysis [1] Key economic factors are the high energy consumption and the competitive price of aluminium - the process must be run continuously and efficiently. To achieve a stable process, the alumina concentration has to be kept in a narrow band, which requires a good alumina feeding strategy and rapid dissolution in the bath. In recent years, the electrolyte volume has decreased (because of reduced anodecathode distance and larger anodes are being used), and the trend has been to increase the amount of aluminium produced per unit time. A deeper and more fundamental understanding of the feeding and dissolution process is needed if further improvements are to be made to the process efficiency.

The industrial process [2] involves several complex steps including the way the alumina is fed into the bath, and the thermochemical reactions between the alumina and the bath. Feeding is typically carried out by breaking the crust that forms at the top of the cell and then pouring alumina as a granular material into the bath, where ideally it

\footnotetext{
* Corresponding author

Email addresses: kovacs@maths.ox.ac.uk (A. Kovács), breward@maths.ox.ac.uk (C.J.W. Breward),

muench@maths.ox.ac.uk (A. Münch), oliver@maths.ox.ac.uk (J.M. Oliver)
}

sinks and dissolves. However, experimental research [3-7] on both the laboratory and industrial scale indicate that, in some circumstances, the alumina particles may form a floating structure of undissolved alumina at the surface of the bath. These rafts may remain afloat for much longer times than the feeding cycle [7], ultimately disrupting the process.

Experimental research $[8,9]$ on the feeding and dissolution process investigates, for example, how the particle dissolution time depends on the bath concentration, the size of the alumina particles, and the initial heating of the particles. Haverkamp and Welch [10] include the effect of stirring of the cryolite bath which accelerates the dissolution by dispersing the alumina particles; this is confirmed by simulations of a mathematical model for the alumina concentration in the bath, and by small sized experiments. An extensive description of various experimental factors influencing the feeding has been covered in a review [2].

Since experiments using molten cryolite are extremely challenging, advanced numerical simulations have proven to be a viable supplement to experiments. For example, Hofer [11] uses a finite element formulation for a model for the Hall-Héroult cell in which the fluid velocity and pressure, the concentration of dissolved alumina, and the position and sizes of the alumina particles are tracked, in order to determine the influence of the flow on the dispersion of both the alumina particles and the dissolved alumina. The finite element framework has also been used by other authors to investigate various aspects of the Hall-Héroult cell [12-14]. In order to be computationally tractable, all 
these simulations rely upon sub-grid models which in turn depend upon closure laws formulated on a rigorous mathematical basis.

In contrast to previous experimental studies, which focus on the dissolution of clumps of alumina particles, we consider the behaviour of a single particle since this will be an important ingredient in the closure laws for bathlevel simulations. The low temperature of the feedstock compared to the bath, which is maintained only slightly above the melting point to save energy, leads to three distinct process stages in the single particle case: first, some of the bath freezes into a shell around the particle; second, this shell melts; third, after the shell has vanished, the alumina particle dissolves into the cryolite. The first two stages are heat-transfer-driven phase changes and are observed in experiments in which porous alumina lumps are inserted into cryolite [3].

Moving boundary problems are ubiquitous in many disciplines such as materials science, geosciences, biology, or even finance. There is a considerable amount of mathematical literature on spherically symmetric moving boundary problems dealing with bubble nucleation [15], bubble dissolution [16], or mass-transfer-controlled dissolution of an isolated sphere [17]. Due to its rich mathematical structure, the inward solidification of a suddenly cooled liquid sphere has been extensively studied for example in [1824]. The analysis in [18] breaks down as the freezing front approaches the centre of the sphere, but this issue was rectified in [19], and improved in [20] by considering the region close to the centre of the sphere when freezing is nearly complete. Similar issues arise in solidification problems in a cylindrical geometry, which was analysed in [22], and for an $N$-dimensional sphere in [21]. In contrast, the previously mentioned models, which are all one-phase, [23, 24] included temperature evolution in both phases, leading to a two-phase Stefan problem. Furthermore, [25] investigated the integral forms of the equations to calculate bounds for the evolution of the radius. Additionally, the coupling of heat transfer and fluid flow is investigated in [26] by analysing the behaviour of a hot fluid flowing over a cold solid plane.

The paper most relevant to our analysis is [27] in which the problem of a metal particle immersed in its melt is considered. They formulate a two-phase Stefan problem similar to our model, with the difference that the contribution of the melt is lumped into the boundary condition using Newton's law of cooling with a constant convective coefficient. We will comment on the differences and similarities as they arise. Our aim in this paper is to formulate and solve a mathematical model to describe the freezing, melting and dissolution process for a single alumina particle, which is an essential building block for understanding alumina feeding and distribution in the Hall-Héroult process.

We will present the mathematical model in Section 2. We then nondimensionalise and analyse the thermal problem in Section 3 and present the analysis for the dissolution problem in Section 4. Finally, we summarise the results and present the conclusions in Section 5.

\section{Mathematical model}

We consider a spherically symmetric geometry in which a solid spherical alumina particle is immersed in a stationary cryolite bath. We suppose that the initial temperature, $T_{p}^{*}$, of the particle is much lower than the liquidus temperature, $T_{m}$, of the cryolite. The cryolite is initially at a temperature $T_{c}^{*}$, which is slightly higher than the liquidus temperature. Thus, a frozen shell of cryolite will form on the surface of the particle, which will then melt away as the temperature equilibrates. We neglect bulk cryolite flow for simplicity and we also neglect the radial flow caused by the phase change during the freezing and melting stages, motivated by the small difference $(1 \%)$ in the densities between frozen and molten cryolite; we revisit this assumption in Section 5.2.

\subsection{Thermal problem}

We denote by $t$ the time since the instantaneous immersion of the particle in the bath and by $r$ the radial coordinate from the centre of the particle. We split the domain into three regions as shown in Figure 1: the solid alumina particle of radius $a$ occupies $0<r<a$; the frozen cryolite shell occupies $a<r<R(t)$, where $R(t)$ is the position of the interface between the frozen and unfrozen region; and finally the molten cryolite resides in $r>R(t)$.

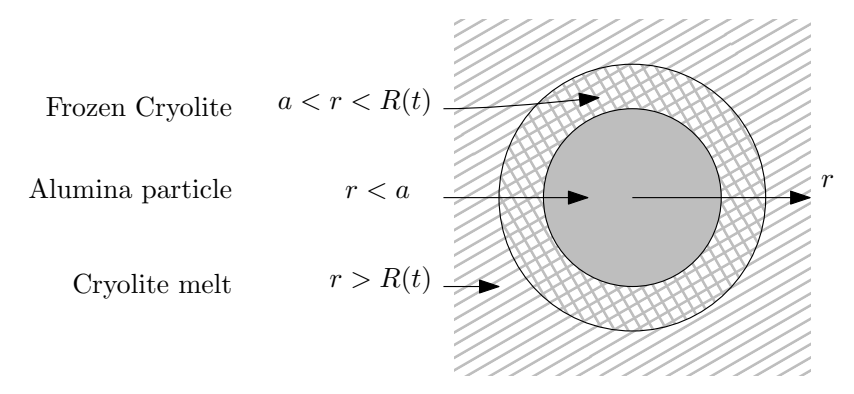

Figure 1: Geometry of the phase-change problem for a spherical particle of radius $a$ with a frozen shell located at $a<r<R(t)$.

Conservation of heat energy and Fourier's law for heat conduction [28] give the governing equations in each region, namely

$$
\begin{aligned}
\rho_{p} c_{p} \frac{\partial T_{p}}{\partial t}=\frac{k_{p}}{r} \frac{\partial^{2}}{\partial r^{2}}\left(r T_{p}\right) & \text { in } & & 0<r<a, \\
\rho_{s} c_{s} \frac{\partial T_{s}}{\partial t}=\frac{k_{s}}{r} \frac{\partial^{2}}{\partial r^{2}}\left(r T_{s}\right) & \text { in } & & a<r<R(t), \\
\rho_{c} c_{c} \frac{\partial T_{c}}{\partial t}=\frac{k_{c}}{r} \frac{\partial^{2}}{\partial r^{2}}\left(r T_{c}\right) & \text { in } & & r>R(t),
\end{aligned}
$$

where $T_{i}(r, t)$ are the temperatures, $\rho_{i}$ are the densities, $c_{i}$ are the specific heat capacities at constant pressure, and $k_{i}$ are the thermal conductivities; here $i \in\{p, s, f\}$, where 
$p$ denotes the particle, $s$ denotes the frozen shell of cryolite and $c$ denotes the molten cryolite.

We assume that the temperature and the heat flux are continuous at the boundary between the particle and the frozen cryolite shell, so that

$$
T_{p}=T_{s}, \quad k_{p} \frac{\partial T_{p}}{\partial r}=k_{s} \frac{\partial T_{s}}{\partial r} \quad \text { on } \quad r=a .
$$

On the moving boundary between the frozen and molten cryolite, $r=R(t)$, we assume that the temperature is continuous and equal to the cryolite liquidus temperature $T_{m}$. We further assume that there is a jump in the thermal flux caused by the phase change. Thus, we write

$T_{s}=T_{c}=T_{m}, \quad k_{s} \frac{\partial T_{s}}{\partial r}-k_{c} \frac{\partial T_{c}}{\partial r}=\rho_{s} L \dot{R} \quad$ on $\quad r=R(t)$,

where $L$ is the latent heat of solidification of the cryolite, which is assumed to be constant, and $\dot{R}=\mathrm{d} R / \mathrm{d} t$. The Stefan condition given in (2.3) is different from the condition used in [27], where they assume Newton's law of cooling at the edge of frozen cryolite rather than modelling the heat transfer in the fluid.

Finally, we assume that the temperature at the centre of the particle is finite, and that the temperature far away from the particle is given, so that

$$
\begin{aligned}
& T_{p}=\mathrm{O}(1) \quad \text { as } \quad r \rightarrow 0, \\
& T_{c} \rightarrow T_{c}^{*} \quad \text { as } \quad r \rightarrow \infty,
\end{aligned}
$$

where $T_{c}^{*}$ is the far-field cryolite temperature, which we note is normally a few degrees above $T_{m}$.

Initially, the temperatures in the particle and the cryolite are spatially homogeneous and there is no frozen shell so that the initial conditions are given by

$$
\begin{aligned}
& T_{p}=T_{p}^{*} \quad \text { for } \quad 0<r<a \text { at } t=0 \text {, } \\
& T_{c}=T_{c}^{*} \quad \text { for } \quad r>a \text { at } t=0 \text {, } \\
& R=a \quad \text { at } t=0 \text {, }
\end{aligned}
$$

where $T_{p}^{*}$ is the initial temperature of the particle (slightly above room temperature). The equations (2.1)-(2.5) form a classical two-phase Stefan problem [24, 36, 37] in the cryolite, with an added region for the particle. We note that, in contrast, the model of [27] is a one-phase Stefan problem involving two domains (particle and shell). We present the key material and physical parameters in Table 1.

We are interested in determining the thickness of the frozen shell. We define the freezing time $t_{F}$ to be the time at which the thickness of the frozen shell is maximal and the melting time $t_{M}$ to be the time at which the frozen shell has melted away to zero thickness. We are also interested in the time of complete dissolution of the particle, denoted by $t_{D}$. A schematic of the process, indicating the timescales, is shown in Figure 2. Our phase change model is only valid up to $t_{M}$, after which the frozen shell region and the moving boundary disappears. After $t_{M}$ we have to solve a standard heat conduction problem in the particle and the cryolite and our interest turns to how the particle dissolves.

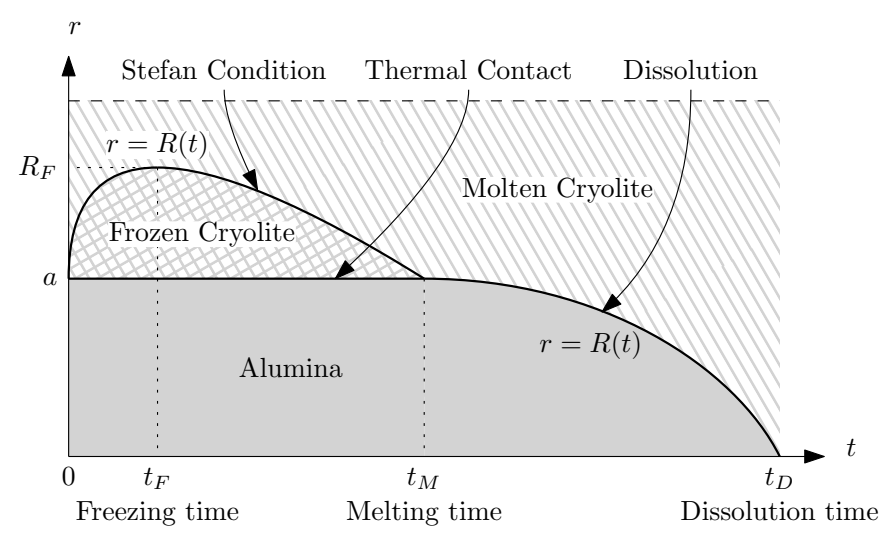

Figure 2: Schematic of the heat conduction and dissolution problems. The freezing model (in Section 2.1) is valid for $0<t<t_{M}$, while the dissolution model (in Section 2.2) is valid for $t_{M}<t<t_{D}$.

\subsection{Dissolution problem}

As soon as the frozen shell has melted, the behaviour of the particle is described by a well-known mass-transfer based problem. The pure dissolution of a particle has been investigated before by e.g. [16, 17], and here we summarise concisely the argument.

The alumina dissolved in the molten cryolite makes up about $4 \%$ (by mass) of the bath, so we define the dilute concentration of alumina in the bath as $C(r, t)$ (with units $\mathrm{kg} \mathrm{m}^{-3}$ ). Assuming spherical symmetry and that the transport of dissolved alumina is due to diffusion and advection, the governing conservation of mass equation is given by

$$
\frac{\partial C}{\partial t}+\frac{1}{r^{2}} \frac{\partial}{\partial r}\left(r^{2} u_{r} C-r^{2} D \frac{\partial C}{\partial r}\right)=0 \quad \text { in } \quad r>R(t),
$$

where $u_{r}(r, t)$ is the radial velocity of the molten cryolite caused by the motion of the edge of the dissolving particle, and $D$ is the alumina diffusivity in the molten cryolite. Assuming that the cryolite has constant density, the velocity field in the molten cryolite satisfies the conservation of mass equation given by

$$
\frac{1}{r^{2}} \frac{\partial}{\partial r}\left(r^{2} u_{r}\right)=0 \quad \text { in } \quad r>R(t)
$$

which can be immediately integrated to give

$$
u_{r}=\frac{A(t)}{r^{2}},
$$

for some function $A(t)$, which we will determine later.

We note that, in this dissolution model, we include the velocity due to the movement of the boundary because the density difference between the alumina and the molten cryolite is substantial (see Table 1). 


\begin{tabular}{|c|c|c|c|}
\hline Parameter & Symbol & Value & Sources \\
\hline \multicolumn{4}{|c|}{ Alumina } \\
\hline initial temperature & $T_{A}$ & $373 \mathrm{~K}$ & {$[29]$} \\
\hline melting temperature & $T_{m}^{A}$ & $2300 \mathrm{~K}$ & {$[30]$} \\
\hline heat conductivity & $k_{p}$ & $4-16 \mathrm{~W} \mathrm{~m}^{-1} \mathrm{~K}^{-1}$ & {$[29,30]$} \\
\hline specific heat capacity & $c_{p}$ & $1200 \mathrm{~J} \mathrm{~kg}^{-1} \mathrm{~K}^{-1}$ & {$[29,30]$} \\
\hline density & $\rho_{p}$ & $2-2.5 \mathrm{~kg} \mathrm{dm}^{-3}$ & {$[29][31$, p. 47$]$} \\
\hline diffusivity in cryolite & $D$ & $1.5 \times 10^{-9} \mathrm{~m}^{2} \mathrm{~s}^{-1}$ & {$[29]$} \\
\hline far-field concentration & $C_{f}$ & $62 \mathrm{~kg} \mathrm{~m}^{-3}$ & {$[29]$} \\
\hline saturation concentration & $C_{s}$ & $165 \mathrm{~kg} \mathrm{~m}^{-3}$ & {$[29]$} \\
\hline \multicolumn{4}{|c|}{ Frozen cryolite } \\
\hline melting point & $T_{m}$ & $1200-1230 \mathrm{~K}$ & {$[31$, p. 43$][32$, p. 52$]$} \\
\hline heat conductivity & $k_{s}$ & $1.5 \mathrm{~W} \mathrm{~m}^{-1} \mathrm{~K}^{-1}$ & {$[29]$} \\
\hline specific heat capacity & $c_{s}$ & $1450 \mathrm{~J} \mathrm{~kg}^{-1} \mathrm{~K}^{-1}$ & {$[29]$} \\
\hline density & $\rho_{s}$ & $2090 \mathrm{~kg} \mathrm{~m}^{-3}$ & {$[29][31$, p.54] } \\
\hline \multicolumn{4}{|c|}{ Molten cryolite } \\
\hline bath temperature & $T_{c}$ & $1223-1243 \mathrm{~K}$ & {$[3,4,33]$} \\
\hline heat conductivity & $k_{c}$ & $0.8 \mathrm{~W} \mathrm{~m}^{-1} \mathrm{~K}^{-1}$ & {$[29]$} \\
\hline specific heat capacity & $c_{c}$ & $1900 \mathrm{~J} \mathrm{~kg}^{-1} \mathrm{~K}^{-1}$ & {$[34]$} \\
\hline density & $\rho_{c}$ & $2070 \mathrm{~kg} \mathrm{~m}^{-3}$ & {$[31$, p. 45$]$} \\
\hline latent heat of solidification & $L$ & $530 \times 10^{3} \mathrm{~J} \mathrm{~kg}^{-1}$ & {$[29]$} \\
\hline dynamic viscosity & $\mu_{c}$ & $2.7 \times 10^{-3} \mathrm{Pas}$ & {$[31$, p. 56$]$} \\
\hline \multicolumn{4}{|c|}{ Environment } \\
\hline typical particle size & $a$ & $50 \times 10^{-6} \mathrm{~m}$ & {$[29][31$, p.65] } \\
\hline agglomerate size & $s$ & $10 \times 10 \times 5 \mathrm{~cm}$ & {$[29]$} \\
\hline top temperature & $T_{t o p}$ & $1000 \mathrm{~K}$ & {$[29]$} \\
\hline addition rate & $Q$ & $1 \mathrm{~kg} \min ^{-1}$ & {$[29]$} \\
\hline porosity & $\phi$ & $0.3-0.45$ & {$[35, \mathrm{~T} 2.1][31, \mathrm{p} .66]$} \\
\hline
\end{tabular}

Table 1: Table showing the key material and physical parameters in the Hall-Héroult process.

At the surface of the particle, we assume that the alumina in the cryolite is in thermodynamic equilibrium with the particle and that the concentration of alumina in the cryolite is equal to a given saturation concentration $C_{s}$. Far away from the particle, we also assume that the concentration is at a known value $C_{f}$. These conditions read

$$
\begin{aligned}
& C=C_{s} \quad \text { on } \quad r=R(t), \\
& C=C_{f} \quad \text { as } \quad r \rightarrow \infty .
\end{aligned}
$$

We note that, in general, the saturation concentration is a function of temperature. However, for simplicity we take it to be a constant.

We have two more conditions due to the moving boundary. They can be derived [15] by considering the conservation of total mass and alumina mass relative to the moving boundary, and are given by

$$
\begin{aligned}
\left(u_{r}-\dot{R}\right) \rho_{c}=-\dot{R} \rho_{p} \quad \text { on } \quad r=R(t), \\
\left(u_{r}-\dot{R}\right) C_{s}-D \frac{\partial C}{\partial r}=-\dot{R} \rho_{p} \quad \text { on } \quad r=R(t),
\end{aligned}
$$

where $\rho_{c}$ is the density of the molten cryolite, $\rho_{p}$ is the density of the alumina particle. Solving (2.11) and (2.12) for the two unknowns, $u_{r}$ and $\dot{R}$, we obtain

$$
\begin{array}{ll}
u_{r}=\dot{R}\left(1-\frac{\rho_{p}}{\rho_{c}}\right) & \text { on } \quad r=R(t), \\
\dot{R}=\frac{D}{\rho_{p}\left(1-C_{s} / \rho_{c}\right)} \frac{\partial C}{\partial r} \quad \text { on } \quad r=R(t) .
\end{array}
$$

We note that these conditions are valid even when the density of the molten cryolite is not uniform. Finally, the initial concentration in the bath and the position of the boundary are given by

$$
\begin{array}{lll}
C=C_{f} \quad \text { in } \quad r>a & \text { at } \quad t=t_{M}, \\
R=a & & \text { at } t=t_{M} .
\end{array}
$$

Before proceeding, we use (2.13) to find $A(t)$ in (2.8) and hence the velocity distribution in the cryolite, which reads

$$
u_{r}=\left(1-\frac{\rho_{p}}{\rho_{c}}\right) \frac{R^{2} \dot{R}}{r^{2}} .
$$

We see that, since the density of the particle $\rho_{p}$ is greater than that of the cryolite, decreasing the radius induces outward flow. 


\section{Analysis of the thermal problem}

\subsection{Nondimensionalisation}

We nondimensionalise (2.1)-(2.5) using the scalings

$$
\begin{gathered}
r=a \tilde{r}, \quad t=\frac{\rho_{p} c_{p} a^{2}}{k_{p}} \tilde{t}, \\
R=a \tilde{R}, \quad T_{i}=T_{p}^{*}+\left(T_{c}^{*}-T_{p}^{*}\right) \tilde{T}_{i} .
\end{gathered}
$$

We note that the temperature scale is chosen so that the dimensionless temperature varies between 0 and 1 initially, while the time scale is chosen to be the diffusive time scale inside the particle. Dropping the tildes, the heat equations in (2.1) become

$$
\begin{array}{rlrrrl}
\frac{\partial T_{p}}{\partial t} & =\frac{1}{r} \frac{\partial^{2}}{\partial r^{2}}\left(r T_{p}\right) & & \text { in } & & 0<r<1, \\
\frac{\partial T_{s}}{\partial t}=\frac{\kappa_{2}}{r} \frac{\partial^{2}}{\partial r^{2}}\left(r T_{s}\right) & \text { in } & & 1<r<R(t), \\
\frac{\partial T_{c}}{\partial t}=\frac{\kappa_{3}}{r} \frac{\partial^{2}}{\partial r^{2}}\left(r T_{c}\right) & \text { in } & & r>R(t) .
\end{array}
$$

The boundary and interface conditions (2.2), (2.3) and (2.4a) become

$$
\begin{array}{llrl}
T_{p}=\mathrm{O}(1) & \text { as } \quad r \rightarrow 0, \\
T_{p}=T_{s}, \quad \beta \frac{\partial T_{p}}{\partial r}=\frac{\partial T_{s}}{\partial r} & \text { on } \quad r=1, \\
T_{c}=T_{s}=\theta_{m}, \frac{\partial T_{s}}{\partial r}-\nu \frac{\partial T_{c}}{\partial r}=\frac{\dot{R}}{\mathrm{St}} & \text { on } \quad r=R, \\
T_{c}=1 & \text { as } \quad r \rightarrow \infty .
\end{array}
$$

The initial conditions (2.5) become

$$
\begin{aligned}
& T_{p}=0 \quad \text { in } \quad 0<r<1 \text { at } t=0 \text {, } \\
& T_{c}=1 \quad \text { in } \quad r>1 \text { at } t=0 \text {, } \\
& R=1 \quad \text { at } t=0 \text {. }
\end{aligned}
$$

The dimensionless parameters are given by

$$
\begin{array}{r}
\kappa_{2}=\frac{\rho_{p} c_{p} k_{s}}{\rho_{s} c_{s} k_{p}}, \quad \kappa_{3}=\frac{\rho_{p} c_{p} k_{c}}{\rho_{c} c_{c} k_{p}}, \quad \theta_{m}=\frac{T_{m}-T_{p}^{*}}{T_{c}^{*}-T_{p}^{*}}, \\
\beta=\frac{k_{p}}{k_{s}}, \quad \nu=\frac{k_{c}}{k_{s}}, \quad \mathrm{St}=\frac{\rho_{p} c_{p}\left(T_{c}^{*}-T_{p}^{*}\right) k_{s}}{\rho_{s} k_{p} L} .
\end{array}
$$

Here, $\kappa_{2}$ is the ratio of thermal diffusivities of the frozen cryolite shell and the alumina particle, $\kappa_{3}$ is the ratio of thermal diffusivities of the molten cryolite and the alumina particle, $\theta_{m}$ is the dimensionless liquidus temperature of the cryolite, $\beta$ is the ratio of the thermal conductivity of alumina to that of the frozen cryolite, $\nu$ is the ratio of the thermal conductivity of the molten cryolite to that of the frozen cryolite, and St is the Stefan number, which is the ratio of the heat required to warm up a unit volume of cryolite from the particle temperature to the cryolite temperature to the heat required to change the phase of a unit volume of cryolite from liquid to solid.

\begin{tabular}{rcc}
\hline Parameter & Sym. & Value \\
\hline Diffusivity ratio & $\kappa_{2}$ & $0.074-0.37(0.13)$ \\
Diffusivity ratio & $\kappa_{3}$ & $0.03-0.15(0.05)$ \\
Melting temperature & $\theta_{m}$ & $0.95-1(0.98)$ \\
Conduction of particle & $\beta$ & $2.7-10.7(7)$ \\
Conduction of melt & $\nu$ & $(0.5)$ \\
Stefan number & $\mathrm{St}$ & $0.19-0.95(0.34)$ \\
Dissolution number & $\sigma$ & 0.05 \\
Density ratio & $\rho_{r}$ & $0.97-1.2(1.1)$ \\
\hline
\end{tabular}

Table 2: Dimensionless parameters, and ranges of values, with mean physical properties written in parentheses, calculated from Table 1.

The typical values for each parameter are given in Table 2, calculated using the data in Table 1 . We see that the Stefan number and the diffusivity ratios, $\kappa_{2}$ and $\kappa_{3}$, are small, while the dimensionless liquidus temperature $\theta_{m}$ is close to 1 .

We will consider three different scenarios. First, we will investigate the model as $t \rightarrow 0$ to gain insight into the early-time behaviour and derive an appropriate initial condition with which to initiate our numerical scheme. We will then use asymptotic analysis to explore the small superheat limit $(\theta-1 \ll 1)$, and the small Stefan number limit $(\mathrm{St} \ll 1)$.

\subsection{Early-time behaviour}

Anticipating that, for early-time, the frozen shell is thin and that temperature changes will be restricted to small regions near the interfaces, we seek the asymptotic self-similar solution given by

$$
T_{i} \sim f_{i}(\eta), \quad R \sim 1+2 \lambda \sqrt{t},
$$

where $\eta=(r-1) / 2 \sqrt{t}=\mathrm{O}(1)$ as $t \rightarrow 0^{+}$, and $\lambda$ is a constant. At leading order as $t \rightarrow 0$ the Stefan problem (3.3)-(3.5) reduces to the similarity formulation given by

$$
\begin{array}{rlrrr}
f_{p}^{\prime \prime}+2 \eta f_{p}^{\prime}=0 & \text { in } & \eta<0, \\
f_{s}^{\prime \prime}+\frac{2 \eta}{\kappa_{2}} f_{s}^{\prime}=0 & \text { in } & 0<\eta<\lambda, \\
f_{c}^{\prime \prime}+\frac{2 \eta}{\kappa_{3}} f_{c}^{\prime}=0 & \text { in } & & \eta>\lambda,
\end{array}
$$

with boundary conditions

$$
\begin{array}{lll}
f_{p} \rightarrow 0 & \text { as } & \eta \rightarrow-\infty, \\
f_{p}=f_{s}, \quad \beta f_{1}^{\prime}=f_{2}^{\prime} & \text { on } & \eta=0, \\
f_{s}=f_{c}=\theta_{m}, \quad f_{2}^{\prime}-\nu f_{3}^{\prime}=\frac{\lambda}{\mathrm{St}} & \text { on } & \eta=\lambda, \\
f_{c} \rightarrow 1 & \text { as } & \eta \rightarrow \infty .
\end{array}
$$

We note that the boundary conditions (3.9a) and (3.9d) follow from matching with outer regions (away from the inner boundary layer regions near $r=1$ ) in which $T_{p}$ and 
$T_{c}-1$ are exponentially small as $t \rightarrow 0$. The solution to (3.8)-(3.9) is given by

$$
\begin{aligned}
f_{p}(\eta) & =\theta_{m} \frac{1+\operatorname{erf}(\eta)}{1+\sqrt{\kappa_{2}} \beta \operatorname{erf}\left(\lambda / \sqrt{\kappa_{2}}\right)} \\
f_{s}(\eta) & =\theta_{m} \frac{1+\sqrt{\kappa_{2}} \beta \operatorname{erf}\left(\eta / \sqrt{\kappa_{2}}\right)}{1+\sqrt{\kappa_{2}} \beta \operatorname{erf}\left(\lambda / \sqrt{\kappa_{2}}\right)} \\
f_{c}(\eta) & =\frac{\left(\theta_{m}-1\right) \operatorname{erf}\left(\eta / \sqrt{\kappa_{3}}\right)+\operatorname{erf}\left(\lambda / \sqrt{\kappa_{3}}\right)-\theta_{m}}{\operatorname{erf}\left(\lambda / \sqrt{\kappa_{3}}\right)-1}
\end{aligned}
$$

in $\eta<0,0<\eta<\lambda$ and $\eta>\lambda$, respectively, with the Stefan condition (3.9c) providing a transcendental equation for $\lambda$, which we find to be

$$
\begin{aligned}
\lambda=\frac{\operatorname{St} \beta \theta_{m}}{\sqrt{\pi}} & \left(\frac{\mathrm{e}^{-\lambda^{2} / \kappa_{2}}}{1+\beta \sqrt{\kappa_{2}} \operatorname{erf}\left(\lambda / \sqrt{\kappa_{2}}\right)}\right. \\
& \left.-\frac{\nu\left(1-\theta_{m}\right)}{\beta \theta_{m} \sqrt{\kappa_{3}}} \frac{\mathrm{e}^{-\lambda^{2} / \kappa_{3}}}{1-\operatorname{erf}\left(\lambda / \sqrt{\kappa_{3}}\right)}\right) .
\end{aligned}
$$

We require $\lambda$ to be positive for the boundary to move outwards initially. This places a restriction on the possible parameter values for which freezing is possible. From (3.11) we find that $\lambda>0$ if

$$
\frac{\sqrt{\kappa_{3}} \beta \theta_{m}}{\nu\left(1-\theta_{m}\right)}>1 \text {. }
$$

In the limit $\mathrm{St} \rightarrow 0$, equation (3.11) gives

$$
\lambda \sim \operatorname{St} \frac{\beta \theta_{m}}{\sqrt{\pi}}\left(1-\frac{\nu\left(1-\theta_{m}\right)}{\sqrt{\kappa_{3}} \beta \theta_{m}}\right) .
$$

We will compare this prediction with the small- $t$ limit of a small-St solution in Section 3.5.

In the limit St $\rightarrow \infty$, we find that $\lambda \rightarrow \lambda_{\infty}$, where $\lambda_{\infty}$ is the unique positive root of the equation

$$
\frac{\beta \theta_{m} \sqrt{\kappa_{3}}}{\nu\left(1-\theta_{m}\right)}=\mathrm{e}^{-\lambda_{\infty}^{2}\left(1 / \kappa_{3}-1 / \kappa_{2}\right)} \frac{1+\beta \sqrt{\kappa_{2}} \operatorname{erf}\left(\lambda_{\infty} / \sqrt{\kappa_{2}}\right)}{1-\operatorname{erf}\left(\lambda_{\infty} / \sqrt{\kappa_{3}}\right)}
$$

and thus, in this limit, the interface speed is independent of St.

In Figure 3 (left) we show a representative early-time solution given by (3.10) and (3.11) for three different (early) times. We see that, as time increases, the temperature in the particle increases and the temperature in the molten cryolite decreases, as expected. We see that a "kink" develops in the temperature profile at the outer edge of the frozen shell because of the jump in the heat flux due to the latent heat requirement. In Figure 3 (right) we show how $\lambda$ varies with the Stefan number and we compare the solution from (3.11) with the asymptotic solutions for small and large Stefan numbers given by (3.13) and (3.14), for three values of $\theta_{m}$. We see excellent agreement in the appropriate limits.

\subsection{Numerical results}

We solve (3.3)-(3.5) numerically using method of lines. We apply boundary fixing transformations given by

$$
r=1+\xi R(t) \quad(0<\xi<1), \quad r=1+R(t)+\zeta \quad(\zeta>0)
$$

in regions $1<r<R(t)$ and $r>R(t)$, respectively. Our spatial discretisation uses central differences and we solve the resulting system of nonlinear ODEs using NDSolve in Wolfram Mathematica 12.0 with the inbuilt adaptive backward differentiation method in time. We initialise the simulation using the asymptotic solutions given by (3.10) and (3.11), setting $t=10^{-4}$. The early-time asymptotics agree with our numerical predictions over at least a decade, as shown in Figure 5. We checked convergence in the usual manner by increasing the spatial resolution and decreasing the error tolerances.

To see the overall behaviour of the model we present results for a range of parameters (in Figures 4-7). We will then present the behaviour for the typical operating parameters given in Table 1 (in Figure 9). In Figure 4, we present the solution for the temperature $T$ and for the shell thickness $R$ in the case in which $\theta_{m}=0.95$, with all other parameters set equal to unity. We see that a frozen shell immediately forms around the particle and that the particle heats up. We see that the cryolite is cooled slightly in a region close to the particle. We also see that the frozen shell thickness reaches a maximum and then begins to thin. When the shell has completely melted, the temperature in the particle is approximately constant.

Next we examine the effect of varying the Stefan number. In Figure 5 (left) we show log-log plots of the frozen shell thickness varying in time for three different Stefan numbers. We note that, motivated by (3.57) which we will derive in Section 3.5, an appropriate scaled shell thickness is given by $3(R-1) /\left(\mathrm{St} \beta \theta_{m}\right)$, which bounds the scaled thickness by 1 . We see that the numerical solutions converge to the small Stefan number solution in the appropriate limit, and that the early-time solution (3.10) and (3.11) holds for multiple decades. We see that there is a small increase in the scaled shell thickness as we decrease the
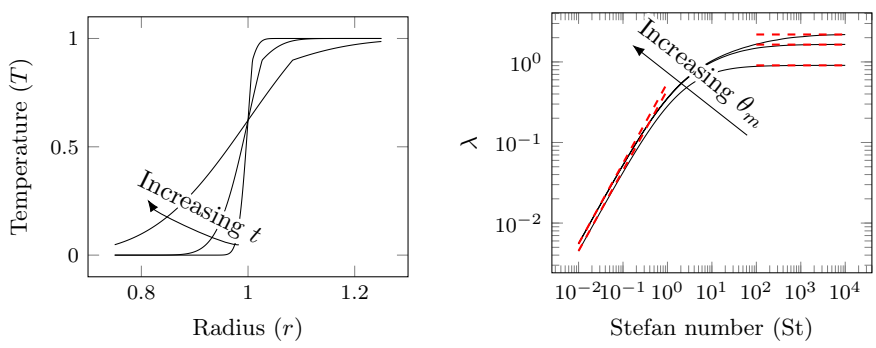

Figure 3: Left: Graph showing how the temperature varies with $r$ given by (3.10) and (3.11) for $t=\left\{10^{-4}, 10^{-3}, 10^{-2}\right\}$. The kink denotes the position of the frozen shell. Right: Graph showing $\lambda$ vs Stefan number given by (3.11) for three different $1-\theta_{m}=$ $\left\{10^{-1}, 10^{-2}, 10^{-3}\right\}$ with all other parameters equal to 1 . Red lines are given by (3.13) and (3.14). 

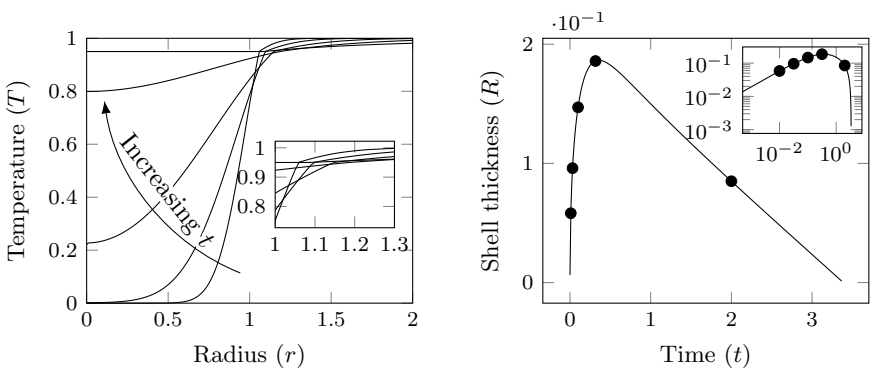

Figure 4: Left: Graph showing the temperature versus radius, given by solving (3.3)-(3.5) numerically at times $t=$ $\left\{10^{-2}, 10^{-1.5}, 10^{-1}, 10^{-0.5}, 2\right\}$. Right: Graph showing how the shell thickness varies with time. The points marked with a circle correspond to the curves in the temperature plot. $\theta_{m}=0.95$; all other parameters set equal to 1 . The inset in the left figure shows a zoom in near $r=1$, meanwhile the inset in the right figure shows the boundary evolution on a logarithmic scale.

Stefan number. However, the actual radius decreases as the Stefan number decreases, which motivates the investigation of the small Stefan number limit.
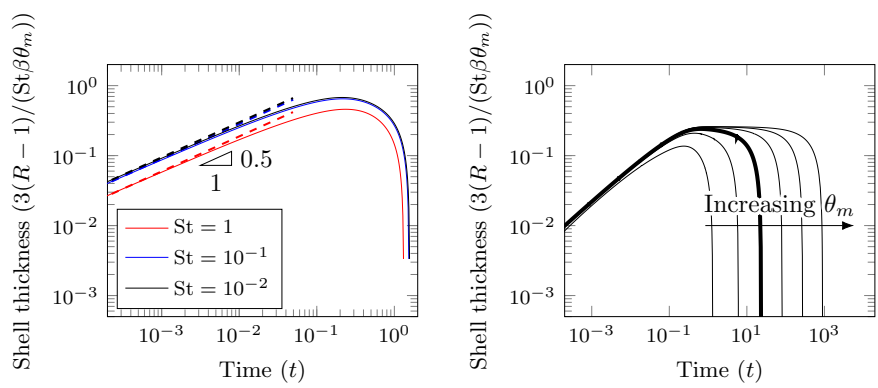

Figure 5: Left: Scaled shell thickness vs time. The solid lines show the numerical solutions given by (3.7) and (3.11) for $\mathrm{St}=$ $10^{-2}, 10^{-1}, 1$; the dashed lines represent the early-time solutions found in Section 3.2. Right: Scaled shell thickness as a function of time for different values of $\theta_{m}$. The lines correspond to logarithmically distributed $1-\theta_{m}$ values between $10^{-4}$ and $10^{-1}$, with all other parameters set equal to 1 . The thick black line corresponds to $\theta_{m}=1$.

In Figure 5 (right), we see that increasing $\theta_{m}$ (the dimensionless liquidus temperature) significantly increases the melting time, introducing a two-timescale behaviour, namely rapid freezing followed by slow melting. In Figure 6 , we see that decreasing $\nu$ has the same effect as increasing $\theta_{m}$; meanwhile increasing $\beta$ significantly increases both the thickness of the frozen shell and the melting time. Physically, these correspond to making the cryolite region non-conductive, decreasing the superheat and making the particle very conductive, respectively.

Finally, we investigate the behaviour of the melting and freezing times, $t_{M}$ and $t_{F}$, as $\theta_{m}$ changes for $\mathrm{St}=1$. In Figure 7, we show graphs of the behaviour as $1-\theta_{m}$ changes. We see that as $\theta_{m} \rightarrow 1^{-}$, the melting time $t_{M}$ scales with $1 /\left(1-\theta_{m}\right)$ (shown as a black line in Figure 7 (left)). We note that the freezing time $t_{F}$ depends only weakly on the superheat, which means that the freezing is
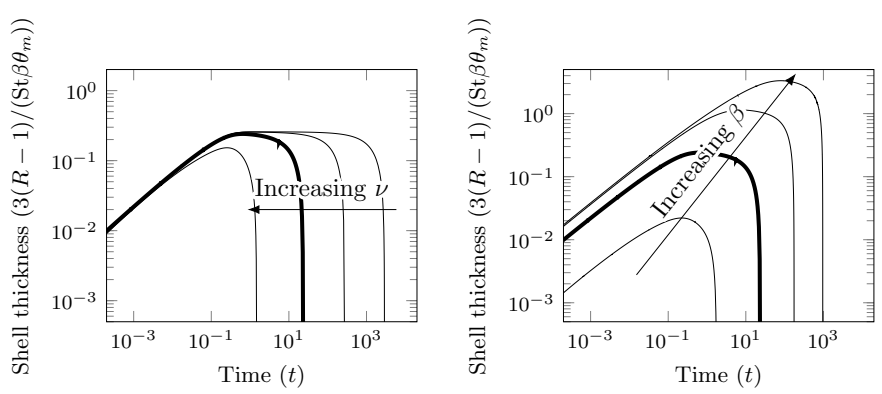

Figure 6: Graphs showing the shell thickness as a function of time for different $\beta$ and $\nu$ values ranging from $10^{-2}$ to $10^{2} . \theta_{m}=0.99$, with all other parameters set equal to 1 .

governed mainly by the time it takes for the temperature to equilibrate inside the particle.

\subsection{Small superheat limit}

As noted earlier, the temperature in the bath is near to the liquidus temperature so that we can write $\theta_{m}=1-\varepsilon$, where $0<\varepsilon \ll 1$. In this limit, motivated by the behaviour in Figure 5 (right), we expect the problem to separate into two different timescales. First, the freezing timescale, in which $t=\mathrm{O}(1)$, and then the melting timescale, in which $t=\mathrm{O}(1 / \varepsilon)$. In the limit $\varepsilon \ll 1$, we expand the dependent variables, and on the freezing timescale have that $T_{c}^{(0)}=1$ and that $T_{p}^{(0)}$ and $T_{s}^{(0)}$ satisfy the same problem as (3.3)(3.5), but with $T_{c}^{(0)}=1$ everywhere in $r>R(t)$. In general there is no analytical solution for this problem. However, we can make progress because we are mainly interested in the steady state the system achieves on the freezing timescale so that we can match to the melting timescale. Crossing out the time derivatives in (3.3a) and (3.3b), we can solve for $\bar{T}_{p}, \bar{T}_{s}$, and $\bar{R}$ where bars denote the steady state versions of $T_{p}, T_{s}$, and $R$. By integrating the righthand side of (3.3a) and (3.3b), and applying the boundary conditions (3.4) we deduce that, in steady state,

$$
\bar{T}_{p}=1 \quad \text { in } \quad 0<r<1, \quad \bar{T}_{c}=1 \quad \text { in } \quad 1<r<\bar{R},
$$

which means that the particle and the shell heats up to the bath temperature, as we would expect intuitively. The leading-order location of the boundary, $\bar{R}$, is determined
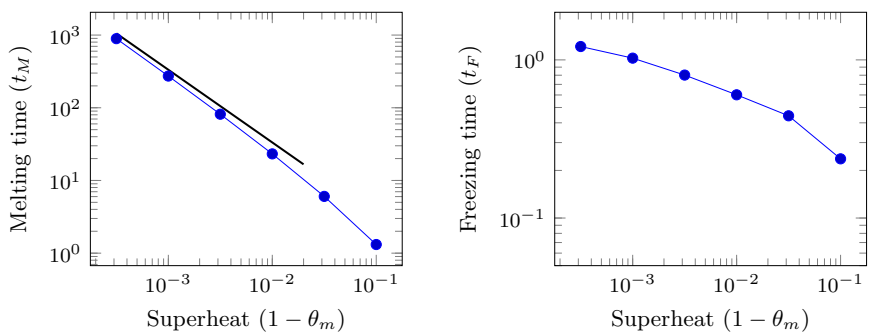

Figure 7: Left: Melting time as a function of the superheat. For small values the behaviour is $t \sim 1 /\left(1-\theta_{m}\right)$ (black line). Right: Freezing time vs superheat. All other parameters are set equal to 1. 
using the total energy of the system. As described in [27] for a similar model, integrating (3.3a) and (3.3b) gives

$\frac{\mathrm{d}}{\mathrm{d} t}\left[\beta \int_{0}^{1} T_{p}^{(0)} r^{2} \mathrm{~d} r+\int_{1}^{R^{(0)}} \frac{T_{s}^{(0)} r^{2}}{\kappa_{2}} \mathrm{~d} r-R^{(0)^{3}} \frac{\frac{1}{\kappa_{2}}+\frac{1}{\mathrm{St}}}{3}\right]=0$.

We can then integrate (3.16) and apply the initial conditions (3.5) and the steady state of the system given by (3.15) to solve for the maximum shell radius $\bar{R}$ as

$$
\bar{R}=\sqrt[3]{1+\beta \mathrm{St}} .
$$

We note that the maximum shell size given by (3.17) is identical to that predicted in [27], which is to be expected since energy is conserved in both models. This solution is, however, only valid for $t \ll 1 / \varepsilon$; on the timescale $t=$ $\mathrm{O}(1 / \varepsilon)$ the boundary shrinks due to heat transfer from the cryolite. To resolve this late-time behaviour, we introduce the scalings

$$
T_{c}=1-\varepsilon \hat{T}_{c}(r, \tau), \quad R=\hat{R}(\tau)
$$

into (3.3)-(3.5) using the long timescale given by

$$
\tau=t \varepsilon=\mathrm{O}(1)
$$

Expanding the dependent variables in powers of $\varepsilon$, the leading-order problem for $\hat{T}_{c}$ is given by

$$
\begin{array}{r}
\varepsilon \frac{\partial \hat{T}_{c}^{(0)}}{\partial \tau}=\frac{\kappa_{3}}{r} \frac{\partial^{2}}{\partial r^{2}}\left(r \hat{T}_{c}^{(0)}\right) \quad \text { in } \quad r>\hat{R}^{(0)}(\tau) \\
\hat{T}_{c}^{(0)}=1 \quad \text { on } \quad r=\hat{R}^{(0)}(\tau) \\
\hat{T}_{c}^{(0)} \rightarrow 0 \quad \text { on } \quad r \rightarrow \infty \\
R=\bar{R} \quad \text { at } \quad \tau=0 \\
\frac{\partial \hat{R}^{(0)}}{\partial \tau}=\nu \frac{\partial \hat{T}_{c}^{(0)}}{\partial r} \text { at } r=\hat{R}^{(0)}(\tau)
\end{array}
$$

where we have used the fact that $T_{s}^{(0)}=1$ (because the particle and the shell temperature are already equilibrated on this longer timescale) in order to simplify (3.24). The leading-order problem can be solved readily to give

$$
\hat{T}_{c}^{(0)}=\frac{\hat{R}^{(0)}(\tau)}{r} .
$$

Substituting (3.25) into (3.24) gives the boundary evolution at long times as

$$
\hat{R}^{(0)}(\tau)=\sqrt{\bar{R}^{2}-2 \nu \tau}
$$

which gives the melting time as

$$
t_{M} \sim \frac{\bar{R}^{2}}{2 \varepsilon \nu} \quad \text { as } \quad \varepsilon=1-\theta_{m} \rightarrow 0^{+}
$$

Hence, we have an expression that is valid for calculating the melting time in the limit of small superheat. Since [27] do not consider superheat, they present no expression for the melting time $t_{M}$, though they do provide expressions in the large diffusivity limit and find that the radius decreases linearly with time.

\subsection{Small Stefan number limit}

In the small Stefan number limit, the thickness of the frozen shell is small (as is shown in Figure 5) because the interface speed found in (3.13) is of $\mathrm{O}(\mathrm{St})$ as $\mathrm{St} \rightarrow 0$, the cryolite being hard to freeze in this limit. From the Stefan condition (3.4c), we see that in this limit $R$ is constant at leading order. Thus, when St $\ll 1$, we introduce a local coordinate in the thin shell by defining

$$
R=1+\operatorname{St} R^{(1)}, \quad r=1+\mathrm{St} \bar{r} .
$$

Substituting (3.28) into (3.3)-(3.5) and assuming that the dependent variables can be expanded in the form $T_{i} \sim$ $T_{i}^{(0)}+\mathrm{St} T_{i}^{(1)}$ as $\mathrm{St} \rightarrow 0$, we find that conduction of heat across the frozen layer is dominant so that, at leading order,

$$
\frac{\partial^{2} T_{s}^{(0)}}{\partial \bar{r}^{2}}=0 \text { for } 0<\bar{r}<R^{(1)},
$$

with leading-order boundary conditions given by

$$
T_{s}^{(0)}=\theta_{m} \quad \text { on } \quad \bar{r}=R^{(1)}, \quad \frac{\partial T_{s}^{(0)}}{\partial \bar{r}}=0 \quad \text { on } \quad \bar{r}=0 .
$$

The solution to (3.29) and (3.30) is simply

$$
T_{s}^{(0)}=\theta_{m},
$$

i.e. the temperature through the frozen shell is equal at leading order to the liquidus temperature. To obtain an equation for the interface position, we have to consider the $\mathrm{O}(\mathrm{St})$ problem in the shell, which reads

$$
\frac{\partial^{2} T_{s}^{(1)}}{\partial \bar{r}^{2}}=0 \text { for } \quad 0<\bar{r}<R^{(1)},
$$

with boundary conditions

$$
\begin{gathered}
T_{s}^{(1)}=0 \quad \text { on } \quad \bar{r}=R^{(1)}, \\
\frac{\partial T_{s}^{(1)}}{\partial \bar{r}}=\left.\beta \frac{\partial T_{p}^{(0)}}{\partial r}\right|_{r=1} \quad \text { on } \quad \bar{r}=0 .
\end{gathered}
$$

The solution for $T_{s}^{(1)}$ is

$$
T_{s}^{(1)}=\left.\beta \frac{\partial T_{p}^{(0)}}{\partial r}\right|_{r=1}\left(\bar{r}-R^{(1)}\right),
$$

and we see from (3.35) that the flux is preserved through the shell and so we can write $(3.4 \mathrm{c})$ as

$$
\dot{R}^{(1)}(t)=\left.\beta \frac{\partial T_{p}^{(0)}}{\partial r}\right|_{r=1^{-}}-\left.\nu \frac{\partial T_{c}^{(0)}}{\partial r}\right|_{r=1^{+}}
$$

with

$$
R^{(1)}=0 \quad \text { at } \quad t=0 .
$$

Thus, at leading-order, the temperatures of the particle and cryolite bath are equal to the liquidus temperature at the boundary, which is linearised onto $R=1$, while the flux of heat out of each of these regions controls the evolution of the thickness of the frozen shell. 
3.5.1. Leading-order solution in the alumina particle

In the particle, the leading-order problem is given by

$$
\frac{\partial T_{p}^{(0)}}{\partial t}=\frac{1}{r} \frac{\partial^{2}}{\partial r^{2}}\left(r T_{p}^{(0)}\right) \quad \text { in } \quad 0<r<1 \quad \text { for } t>0,
$$

with the boundary and initial conditions given by

$$
\begin{aligned}
& T_{p}^{(0)} \text { bdd as } r \rightarrow 0 \text { for } t>0 \text {, } \\
& T_{p}^{(0)}=\theta_{m} \quad \text { on } \quad r=1 \quad \text { for } \quad t>0 \text {, } \\
& T_{p}^{(0)}=0 \quad \text { in } \quad 0 \leq r \leq 1 \quad \text { at } \quad t=0 .
\end{aligned}
$$

Noting that $r T_{p}^{(0)}$ satisfies a one-dimensional problem in Cartesian variables, we can use Fourier theory to find that the solution is given by

$$
T_{p}^{(0)}(r, t)=\theta_{m}\left(1+2 \sum_{j=1}^{\infty} \frac{(-1)^{j}}{j \pi r} \sin (j \pi r) \mathrm{e}^{-j^{2} \pi^{2} t}\right) .
$$

Hence, the heat flux at the boundary is given by

$$
\left.\beta \frac{\partial T_{p}^{(0)}}{\partial r}\right|_{r=1^{-}}=2 \beta \theta_{m} \sum_{j=1}^{\infty} \mathrm{e}^{-j^{2} \pi^{2} t} \quad \text { for } \quad t<t<t_{M} .
$$

The infinite sum is a strictly decreasing function of time, which tends to 0 as $t \rightarrow \infty$. As $t \rightarrow \infty, T_{p}^{(0)} \rightarrow \theta_{m}$ although we note that the solution is only physically relevant for $t<t_{M}$. For early-times, motivated by [24, Eq. 2.12] we use the transformation given by

$$
w(z)=\sum_{n=1}^{\infty} \mathrm{e}^{-n^{2} \pi z}, \quad 1+2 w(z)=z^{-1 / 2}(1+2 w(1 / z)),
$$

to rewrite the right-hand side of (3.41) using the fact that it is equal to $2 \beta \theta_{m} w(\pi t)$; hence as $t \rightarrow 0$, the heat flux is more conveniently written as

$$
\left.\beta \frac{\partial T_{p}^{(0)}}{\partial r}\right|_{r=1^{-}}=\beta \theta_{m}\left(-1+\frac{1}{\sqrt{\pi t}}\left(1+2 \sum_{j=1}^{\infty} \mathrm{e}^{-j^{2} / t}\right)\right) .
$$

Thus, the leading-order behaviour of the heat flux at small times is given by

$$
\left.\beta \frac{\partial T_{p}^{(0)}}{\partial r}\right|_{r=1^{-}} \sim \frac{\beta \theta_{m}}{\sqrt{\pi t}} \quad \text { as } \quad t \rightarrow 0 .
$$

\subsubsection{Leading-order solution in the molten cryolite}

The leading-order problem for $T_{c}^{(0)}$ is given by

$$
\frac{\partial T_{c}^{(0)}}{\partial t}=\frac{\kappa_{3}}{r} \frac{\partial^{2}}{\partial r^{2}}\left(r T_{c}^{(0)}\right) \quad \text { in } \quad r>1 \text { for } t>0 \text {, }
$$

with the following boundary and initial conditions

$$
\begin{aligned}
& T_{c}^{(0)}=\theta_{m} \quad \text { on } \quad r=1 \quad \text { for } \quad t>0 \text {, } \\
& T_{c}^{(0)} \rightarrow 1 \quad \text { as } \quad r \rightarrow \infty \quad \text { for } t>0 \text {, } \\
& T_{c}^{(0)}=1 \quad \text { in } \quad r \geq 1 \quad \text { at } \quad t=0 .
\end{aligned}
$$

This problem admits the similarity solution given by

$$
T_{c}^{(0)}(r, t)=1+\frac{\left(1-\theta_{m}\right)\left(\operatorname{erf}\left(\frac{r-1}{2 \sqrt{\kappa_{3} t}}\right)-1\right)}{r},
$$

for $r>1$ and $0<t<t_{M}$.

The flux through the phase boundary $r=1$ is

$$
\nu \frac{\partial T_{c}^{(0)}}{\partial r}\left(1^{+}, t\right)=\nu\left(1-\theta_{m}\right)\left(\frac{1}{\sqrt{\kappa_{3} \pi t}}+1\right) \quad \text { for } \quad t>0 .
$$

Since all the parameters are positive and $\theta_{m}<1$, the heat flux is positive for all time with

$$
\nu \frac{\partial T_{c}^{(0)}}{\partial r}\left(1^{+}, t\right) \sim \nu \frac{1-\theta_{m}}{\sqrt{\kappa_{3} \pi t}} \quad \text { as } \quad t \rightarrow 0 .
$$

In Figure 8, we show the temperature distributions in the particle and in the cryolite, found by solving (3.3)(3.5), for various times while the frozen region exists. We see from (3.40) that the particle warms up and the cryolite cools, as expected.

We note that if we move away from the boundary by 510 particle diameters, the cryolite temperature is close to the undisturbed bath temperature. This suggests a critical length scale which distinguishes between well distributed and closely packed particles.

\subsubsection{Motion of the freezing surface}

Now that we have expressions for the fluxes, namely (3.43) and (3.48), we can substitute them into the Stefan condition (3.37), to find that

$$
\dot{R}^{(1)}(t)=2 \beta \theta_{m} \sum_{j=1}^{\infty} \mathrm{e}^{-\pi^{2} j^{2} t}-\nu\left(1-\theta_{m}\right)\left(\frac{1}{\sqrt{\pi \kappa_{3} t}}+1\right) .
$$

We integrate (3.50) and apply the initial condition $R_{1}=0$ to obtain

$$
R^{(1)}=\beta \theta_{m}\left(\frac{1}{3}-2 \sum_{j=1}^{\infty} \frac{\mathrm{e}^{-\pi^{2} j^{2} t}}{\pi^{2} j^{2}}\right)-\nu\left(1-\theta_{m}\right)\left(t+\frac{2 \sqrt{t}}{\sqrt{\pi \kappa_{3}}}\right) .
$$
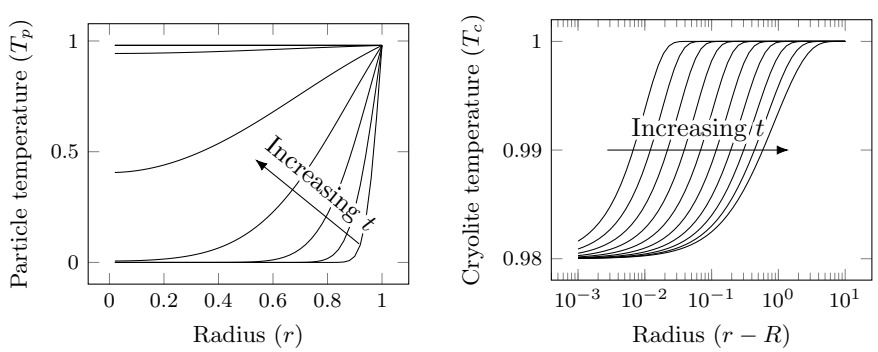

Figure 8: Graphs showing the temperature distribution in the particle given by equation (3.40) (on left) and cryolite given by equation (3.47) (on right) at different times (uniformly logarithmically distributed between $t=10^{-3}$ and $t=164$ ). The parameters are $\beta=7$, $\nu=0.5, \kappa_{3}=0.05$, and $\theta_{m}=0.98$. Note that both figures have the same number of lines at the same times, but on the left multiple lines collapse onto the spatially uniform state. 
We plot $R_{1}(t)$ in Figure 9. We see that the frozen shell grows on a short timescale, reaches a maximum and then melts away again over a longer timescale. Using (3.50) and (3.51), we find $t_{F}$ and $t_{M}$ are given implicitely by

$$
0=2 \beta \theta_{m} \sum_{j=1}^{\infty} \mathrm{e}^{-\pi^{2} j^{2} t_{F}}-\nu\left(1-\theta_{m}\right)\left(\frac{1}{\sqrt{\pi \kappa_{3} t_{F}}}+1\right),
$$

$0=\beta \theta_{m}\left(\frac{1}{3}-2 \sum_{j=1}^{\infty} \frac{\mathrm{e}^{-\pi^{2} j^{2} t_{M}}}{\pi^{2} j^{2}}\right)-\nu\left(1-\theta_{m}\right)\left(t_{M}+\frac{2 \sqrt{t_{M}}}{\sqrt{\pi \kappa_{3}}}\right)$.

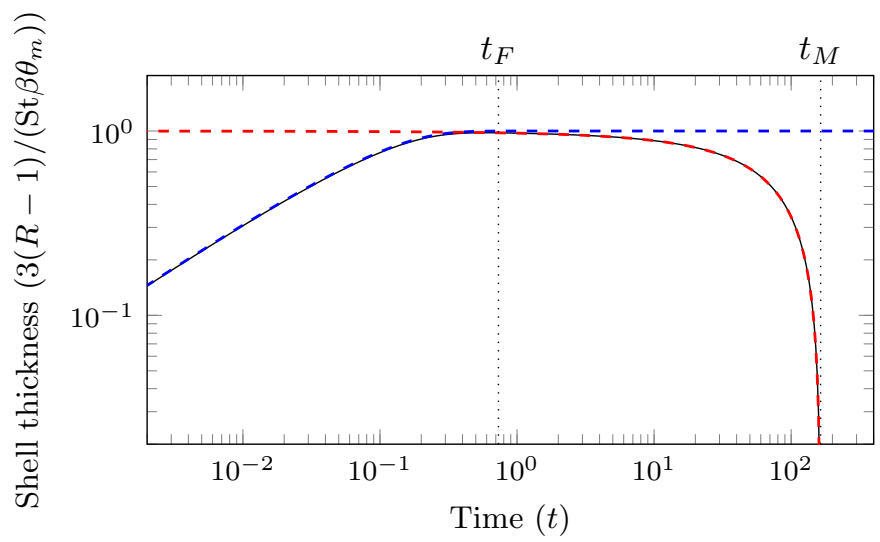

Figure 9: Graph showing the scaled shell thickness given by (3.51) as a function of time (black). Fast freezing given by (3.56) (blue) tends to a constant value given by (3.57), while the slow melting effect given by (3.60) (red) can be used to provide an approximation for the melting time given by (3.61) as $\theta_{m} \rightarrow 1 . t_{F}$ is given by (3.65). The parameters are $\beta=7, \nu=0.5, \kappa_{3}=0.05, \theta_{m}=0.98$.

If we consider the special case $\theta_{m}=1$, in which the bath is everywhere at the liquidus temperature, then the second term in (3.51) disappears and the liquid will freeze and form a shell which will grow until its thickness reaches $\beta / 3$. On the other hand, if $\theta_{m}=0$, the alumina particle is added at the liquidus temperature of the cryolite, and the molten cryolite will not freeze - the particle will simply heat up to the temperature of the bath as seen in Section 3.4.

It is useful to rearrange the terms in (3.50) to further investigate the behaviour by writing

$$
\frac{3 \dot{R}^{(1)}}{\beta \theta_{m}}=6 \sum_{j=1}^{\infty} \mathrm{e}^{-\pi^{2} j^{2} t}-3 \delta\left(\frac{1}{\sqrt{\pi \kappa_{3} t}}+1\right),
$$

with

$$
\delta=\frac{\nu\left(1-\theta_{m}\right)}{\beta \theta_{m}} .
$$

Substituting in the parameters in Table 1 , we find that $\delta \approx 10^{-3}$ and that the solution to (3.54) in the limit $\delta \ll 1$, with initial condition given by $R^{(1)}=0$, is

$$
\frac{3 R^{(1)}}{\beta \theta_{m}}=1-\frac{6}{\pi^{2}} \sum_{j=1}^{\infty} \frac{\mathrm{e}^{-\pi^{2} j^{2} t}}{j^{2}} .
$$

This gives us the maximum radius in the large-time limit of the $t=\mathrm{O}(1)$ problem as

$$
R^{(1)}=\frac{\beta \theta_{m}}{3},
$$

and thus we predict that

$$
R_{\max } \sim 1+\frac{\mathrm{St} \beta \theta_{m}}{3} \quad \text { as } \quad \delta \rightarrow 0, \quad \text { St } \rightarrow 0 .
$$

This expression is the same if we take the small Stefan number limit of (3.26). For large time, we rescale $t=$ $T / 3 \delta$, so that (3.54) becomes, at leading order in $\delta$,

$$
\frac{3}{\beta \theta_{m}} \frac{\mathrm{d} R^{(1)}}{\mathrm{d} T}=-1
$$

We solve (3.59) and match with the long-time behaviour of the shorter time problem, to find that

$$
\frac{3 R^{(1)}}{\beta \theta_{m}}=1-T
$$

which gives us an explicit estimate for the melting time as

$$
t_{M}=\frac{1}{3 \delta}=\frac{\beta \theta_{m}}{\nu\left(1-\theta_{m}\right)},
$$

which is consistent with (3.27). For $t \ll 1$ the behaviour of $(3.50)$ is

$$
\dot{R}^{(1)} \sim \frac{\theta_{m} \beta}{\sqrt{\pi t}}\left(1-\frac{\nu}{\beta \sqrt{\kappa_{3}}} \frac{1-\theta_{m}}{\theta_{m}}\right)>0 \quad \text { as } \quad t \rightarrow 0,
$$

where again we have made use of (3.42). Since all the parameters are positive, in order for the cryolite to freeze, we require

$$
\frac{\sqrt{\kappa_{3}} \beta \theta_{m}}{\nu\left(1-\theta_{m}\right)}>1, \quad \frac{\sqrt{\kappa_{3}}}{\delta}>1
$$

We find a simple upper bound for the freezing time by noticing that the cryolite temperature distribution results in a constant steady-state heat flux. As the sum in (3.51) rapidly decreases, we balance

$$
\nu\left(1-\theta_{m}\right) \approx 2 \beta \theta_{m} \mathrm{e}^{-\pi^{2} t},
$$

which yields

$$
t_{F} \approx \frac{1}{\pi^{2}} \log \left(\frac{2}{\delta}\right)
$$

We note that, in (3.63) and (3.65), the main parameters are $\kappa_{3}$ (see (3.6)) and $\delta$ which represents the ratio of the heat transfer rates in the cryolite and the alumina particle, and describes how intensive the heat flow is between the regions. If $\delta$ is small, the timescale for solidification is much smaller than the melting and so we expect a shell to form. Conversely, if $\delta$ is large then the temperatures equilibrate fast and no freezing is possible.

In Figure 10, we compare the numerical solutions of the full system (3.3)-(3.5) with the asymptotic solutions given by (3.51). We see that the numerical solutions converge to the small Stefan number solution in the appropriate limit. 


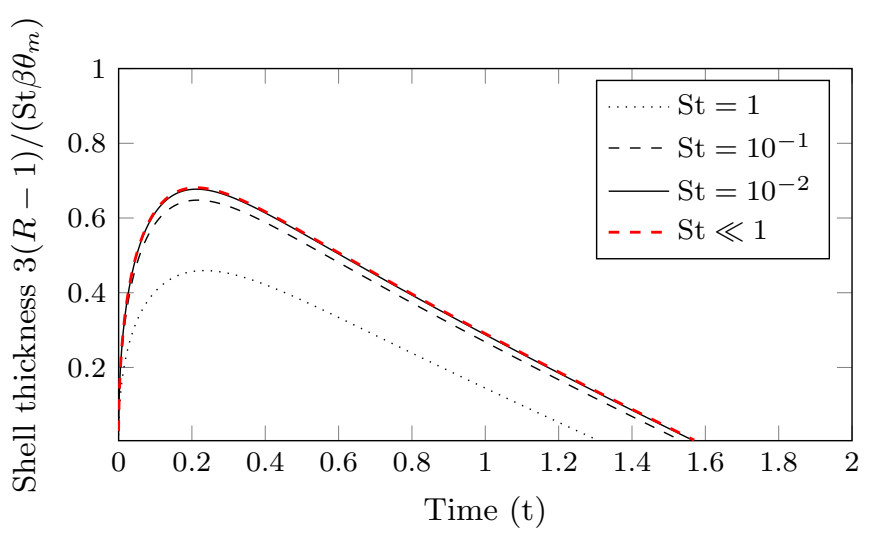

Figure 10: Graph showing the scaled shell thickness as a function of time for different Stefan numbers The black curves correspond to numerical solutions and the small-St limit in red from Section 3.5.

\section{Analysis of the dissolution problem}

\subsection{Nondimensionalisation}

Since we assume that dissolution can only start after complete melting of the frozen shell, we nondimensionalise $^{1}$ the system $(2.6),(2.10)$ and (2.14)-(2.16) using the scalings

$$
\begin{gathered}
r=a \hat{r}, \quad R=a \hat{R}, \quad t-t_{M}=\frac{a^{2}}{D \sigma} \tau, \\
C=C_{f}+\left(C_{s}-C_{f}\right) \hat{C} .
\end{gathered}
$$

Eliminating the liquid velocity, the full dimensionless system can be written as

$$
\frac{\partial C}{\partial \tau}=\frac{1}{r^{2}} \frac{\partial}{\partial r}\left(\frac{r^{2}}{\sigma} \frac{\partial C}{\partial r}-\left(1-\rho_{r}\right) R^{2} \frac{\mathrm{d} R}{\mathrm{~d} \tau} C\right) \quad \text { in } \quad r>R(\tau),
$$

with

$$
\begin{aligned}
& C=1, \quad \frac{\mathrm{d} R}{\mathrm{~d} \tau}=\frac{\partial C}{\partial r} \quad \text { on } \quad r=R(\tau), \\
& C \rightarrow 0 \quad \text { as } r \rightarrow \infty, \\
& C=0 \quad \text { in } r>R(\tau) \text {, } \\
& R=1 \quad \text { at } \tau=0 \text {, }
\end{aligned}
$$

where we have introduced the dimensionless parameters

$$
\sigma=\frac{C_{s}-C_{f}}{\rho_{p}\left(1-C_{s} / \rho_{c}\right)}, \quad \rho_{r}=\frac{\rho_{p}}{\rho_{c}},
$$

where $\sigma$ is the dissolution Stefan number, and $\rho_{r}$ is the density ratio of the alumina to the cryolite. We see in Table 2 that $\sigma=0.05$ and $\rho_{r}=2$. We will now investigate the early-time behaviour of (4.3) analytically, then numerically in Section 4.3 and finally in the small- $\sigma$ limit in Section 4.4.

\footnotetext{
${ }^{1}$ It is worth noting that there are two natural choices for the timescale: the dissolution timescale that we used in (4.1), which balances the two terms in the second boundary condition in (2.14), and the diffusive timescale $a^{2} / D$, representing the balance in the governing equation (4.3a).
}

\subsection{Early-time behaviour}

To initiate our the numerical simulations, it is necessary to derive the early-time behaviour (as $C$ is discontinuous initially at $r=R$ ). The analysis is similar to that for the temperature problem presented in Section 3.2. The concentration is exponentially small except in a boundary layer in which we find that the asymptotic similarity solution is given by

$$
C \sim f(\eta), \quad R \sim 1-2 \lambda \sqrt{\tau} / \sqrt{\sigma}
$$

with $\eta=\sqrt{\sigma}(r-1) /(2 \sqrt{\tau})=\mathrm{O}(1) \quad$ as $\quad \tau \rightarrow 0^{+}$, where $\lambda$ is a constant that needs to be found as part of the solution. After substituting (4.5) into (4.3) and expanding for small $\tau$, we find that the leading-order equation for $f$ is given by

$$
2 \eta f^{\prime}(\eta)+f^{\prime \prime}(\eta)=0
$$

while the boundary and matching conditions are given by

$$
f(-\lambda)=1, \quad f(\eta) \rightarrow 0 \quad \text { as } \quad \eta \rightarrow \infty .
$$

The solution to (4.6) and (4.7) is

$$
f(\eta)=\frac{1-\operatorname{erf}(\eta)}{1+\operatorname{erf}(\lambda)}
$$

where $\lambda$ is the unique positive root of the transcendental equation

$$
\lambda \sqrt{\pi}(1+\operatorname{erf}(\lambda))=\sigma \mathrm{e}^{-\lambda^{2}} .
$$

Equation (4.8) is often referred to as the Neumann Solution. The asymptotic behaviour of $\lambda$ for small and large $\sigma$ are given by

$$
\begin{gathered}
\lambda \sim \sigma / \sqrt{\pi} \quad \text { as } \quad \sigma \rightarrow 0, \\
\lambda \sim \log ^{1 / 2}(\sigma / \sqrt{2 \pi}) \quad \text { as } \quad \sigma \rightarrow \infty .
\end{gathered}
$$

These limits are shown in Figure 11, along with the solution to (4.9), showing excellent agreement in both limits.

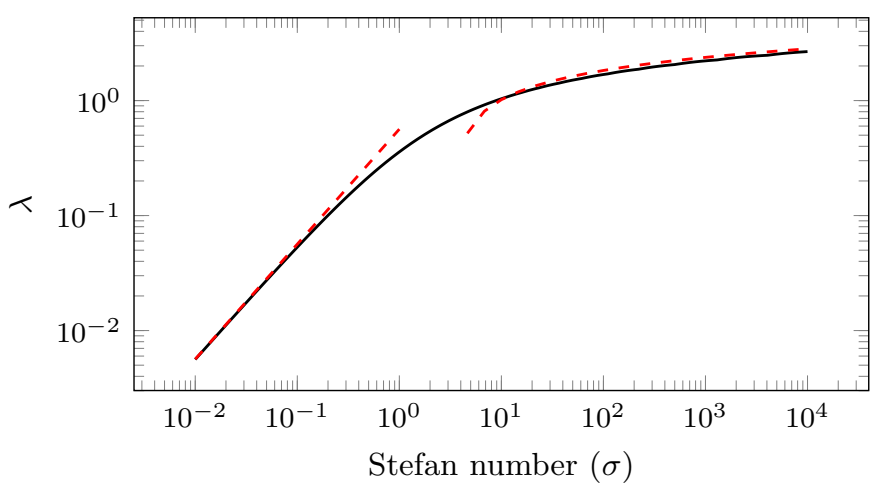

Figure 11: Graph showing $\lambda$ given by (4.9) as $\sigma$ varies, along with the two asymptotic limits (4.11). 


\subsection{Numerical results}

We use (4.5), (4.8) and (4.9) to initiate our numerical simulations to (4.3). We solve the model by the method of lines using a discretisation analogous to the one described in Section 3.3. We show how the radius of the particle varies with time for various values of $\sigma$ in Figure 12 (left). We see that, for small $\sigma$, the numerical results agree with the small- $\sigma$ asymptotic result that we will derive in (4.14). For large $\sigma$, we see that the numerical results agree with the small-time solution presented in (4.5) and (4.11).
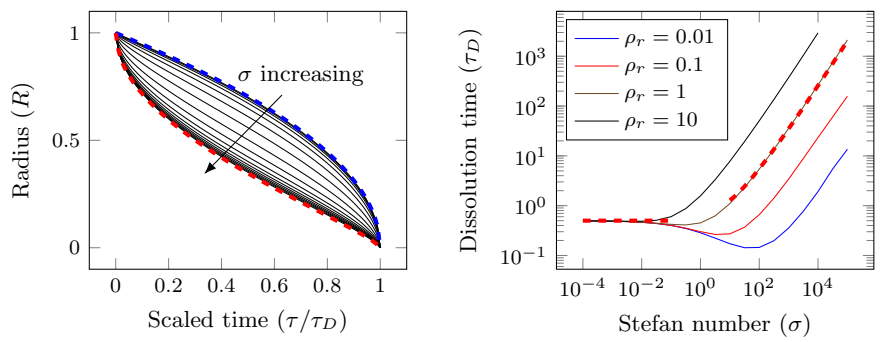

Figure 12: Left: $R(\tau)$ for varying $\sigma$ for $\rho_{r}=1$. The lines range between $\sigma=\left\{10^{-4}, 10^{5}\right\}$. The red dashed line is the small-time solution, while the blue dashed line is the small- $\sigma$ solution given by (4.14). Right: Dissolution time for varying Stefan number $(\sigma)$ for different $\rho_{r}$ values showing the non-monotonicity at $\sigma \approx 1 / \rho_{r}$. Note that the two asymptotic limits are denoted with dashed red lines.

The dissolution time is also an important prediction of our model. In Figure 12 (right), we plot the dissolution time for different $\sigma$ values. We note that, in the small- $\sigma$ limit, the dissolution time $t_{D}$ tends to a constant, while the behaviour for large $\sigma$ is more complicated. At first sight the scaling looks like $\sigma$, however $\sigma / \log (\sigma)$ (shown with a thick black line in Figure 12 (right)) looks like a more appropriate candidate, hinting at logarithmic behaviour in this limit. The final observation that we make is that the $t_{D^{-}} \sigma$ curve is non-monotonic when $\rho_{r}<1$; there is a minimum dissolution time which occurs when $\sigma \approx 1 / \rho_{r}$.

In Figure 12, we also see that, in the small- $\sigma$ limit, the dependence on the density disappears. Physically this means that, if the boundary is moving slowly, then the induced fluid movement due to the phase change is also small and the density ratio only changes the higher-order dynamics.

\subsection{Small- $\sigma$ limit}

In this section, we will consider the physically relevant limit of small $\sigma$ by expanding

$$
R \sim R^{(0)}+\sigma R^{(1)}, \quad C \sim C^{(0)}+\sigma C^{(1)} \quad \text { as } \quad \sigma \rightarrow 0 .
$$

A regular perturbation analysis shows that the leadingorder solution is quasi-steady with (4.3) giving

$$
C^{(0)}=\frac{R^{(0)}}{r}, \quad \frac{\mathrm{d} R^{(0)}}{\mathrm{d} \tau}=-\frac{1}{R^{(0)}} ;
$$

applying the initial condition $R^{(0)}(0)=1$ then gives

$$
R^{(0)}=\sqrt{1-2 \tau} .
$$

We can see from (4.14) that complete dissolution occurs at $\tau_{D} \sim 1 / 2$ as $\sigma \rightarrow 0$, after which the particle ceases to exist. This result has been calculated by many authors, e.g. [17]. By undoing the nondimensionalisation, we see that the leading-order dissolution time $t_{D}$ is given by

$$
t_{D}-t_{M} \sim \frac{a^{2}}{2 D \sigma}=\frac{a^{2} \rho_{p}\left(1-C_{s} / \rho_{c}\right)}{2 D\left(C_{s}-C_{f}\right)} \quad \text { as } \quad \sigma \rightarrow 0 .
$$

\section{Conclusions}

\subsection{Summary of scales}

In Figure 13, we plot $t_{F}, t_{M}$ and $t_{D}$ given by (3.52), (3.53) and (4.15) as a function of $\beta \theta_{m} \sqrt{\kappa_{3}} / \nu\left(1-\theta_{m}\right)$, where $\delta$ is defined in (3.55). There are two qualitatively different regimes. Below the freeze limit (shown by the vertical dotdashed line given by (3.12)) the particle simply dissolves, since the heat transfer in the cryolite is fast enough that the cryolite temperature remains above the freezing temperature and no freezing occurs. However, above this limit freezing, melting, and dissolution all occur. The actual operating regime is shown as a vertical dotted line. We also see that the dissolution takes the largest amount of time, except in the limit $\delta \rightarrow 0$ corresponding to $\theta_{m} \rightarrow 1$, where the melting takes over. A similar behaviour is shown by [26] for the related problem of turbulent flow of lava over a cold surface.

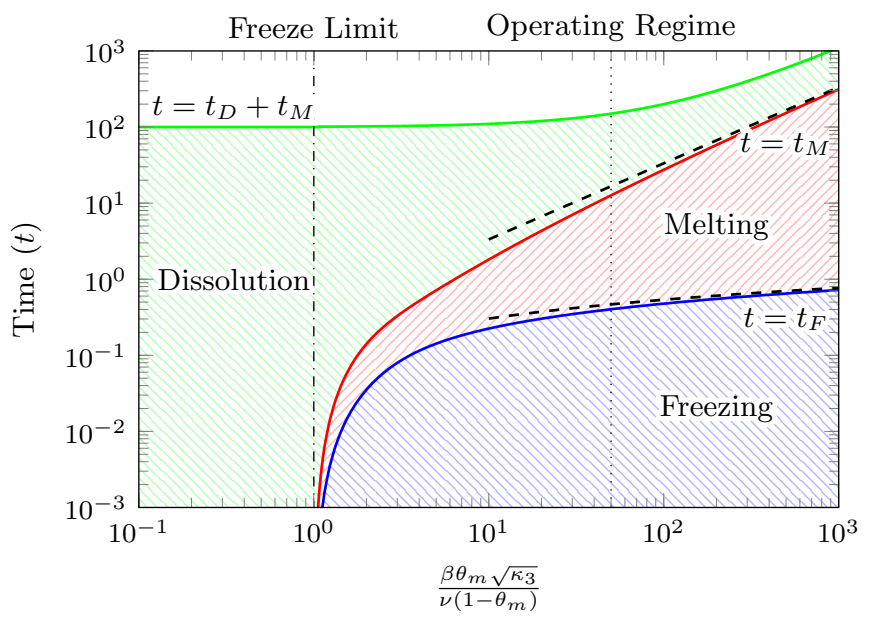

Figure 13: Graph showing $t_{F}, t_{M}$ and $t_{D}$ given by (3.52), (3.53) and (4.15) as a function of $\beta \theta_{m} \sqrt{\kappa_{3}} / \nu\left(1-\theta_{m}\right)$, coloured blue, red and green, respectively. The parameters are St $=0.34, \beta=7, \nu=$ $0.5, \kappa_{3}=0.05$, and $\theta_{m}=0.98$. Dashed lines are given by (3.61) and (3.65) and are valid as $\delta \rightarrow 0$. The dotted vertical line indicate the operating regime of the Hall-Héroult cell, and the freeze limit is given by (3.12).

We use the data to obtain analytical predictions for the dimensional freezing time $t_{F}$, melting time $t_{M}$, and dissolution time $t_{D}$ for a $50 \mu \mathrm{m}$ particle, which we find to be

$$
t_{F} \approx \frac{a^{2} c_{p} \rho_{p}}{\pi^{2} k_{p}} \log \left(\frac{2\left(T_{m}-T_{p}^{*}\right) k_{p}}{\left(T_{c}^{*}-T_{m}\right) k_{c}}\right) \approx 500 \mu \mathrm{s}
$$


from (3.65),

$$
t_{M} \sim \frac{a^{2} c_{p} \rho_{p}}{3 k_{p}} \frac{\left(T_{m}-T_{p}^{*}\right) k_{p}}{\left(T_{c}^{*}-T_{m}\right) k_{c}} \approx 100 \mathrm{~ms},
$$

from (3.61) and

$$
t_{D} \sim \frac{a^{2} \rho_{p}\left(1-C_{s} / \rho_{c}\right)}{2 D\left(C_{s}-C_{f}\right)} \approx 17 \mathrm{~s},
$$

from (4.15). The ratios of these three timescales are independent of $a$ and, for the parameters given in Table 1, are $t_{D} / t_{M} \approx 170$ and $t_{M} / t_{F} \approx 200$. We note that the approximations for the melting and freezing times are only strictly valid when the liquidus temperature is close to the cryolite temperature, i.e. $1-\theta_{m}$ is small (as is true in our case), and that our results only depend on one property of the frozen cryolite, namely the liquidus temperature $T_{m}$. We also note that the dissolution timescale $t_{D}$ is a factor of 10 smaller than that observed by Haverkamp and Welch [10], which is likely due to the difference between dissolving one particle rather than dissolving a powder.

\subsection{Bulk flow}

We now revisit our modelling assumption concerning the neglect of the bulk flow. If we consider a stationary solid alumina particle of radius $a$ in a background cryolite flow with average speed $U$, with dynamic viscosity $\mu_{c}$, then the particle Reynolds number is given by

$$
\operatorname{Re}=\frac{a U \rho_{c}}{\mu_{c}} .
$$

Assuming a bulk velocity of $10 \mathrm{~cm} \mathrm{~s}^{-1}$ and using the other parameters in Table 1 , we find that $\operatorname{Re} \approx 4$, which means that the particle is in the "Stokes drag" regime. The dimensionless governing equation for the location $\mathbf{r}$ of the particle, assuming Stokes drag, is

$$
\operatorname{Stk} \ddot{\mathbf{r}}=\mathbf{u}-\dot{\mathbf{r}}-\mathrm{G} \mathbf{k},
$$

where $\mathbf{u}$ is the velocity of the cryolite and $\mathbf{k}$ is the unit vector in the vertical direction and where

$$
\begin{gathered}
\text { Stk }=\frac{2 \rho_{p} U a^{2}}{9 \mu_{c} H} \approx 10^{-5}, \\
\mathrm{G}=\frac{2\left(\rho_{p}-\rho_{c}\right) g a^{2}}{9 U \mu_{c}} \approx 10^{-3} .
\end{gathered}
$$

Since both Stk and G are small, we conclude that both inertia and gravity can be neglected compared to drag, and hence the particles follow the flow. This also means that the relative velocity between the flow and particle is small, so we are justified in neglecting the influence of flow on the freezing, melting and dissolution, and hence we are permitted to use spherically symmetric geometry. These assumptions break down when Stk and $G$ are $O(1)$, which occurs for particles with millimetre or bigger radii.

\subsection{Summary}

In this article, we have examined the problem of adding a cold alumina particle to a bath of hot cryolite. We developed a spherically symmetric model to describe heat and mass transfer between the particle and the surrounding material, allowing for freezing and melting of the cryolite close to the particle and its subsequent dissolution. We solved this model numerically using the method of lines by using the early-time asymptotic solution to initiate the numerical scheme. We explored the behaviour as we varied the key dimensionless parameters. We also considered the problem in the limits of small superheat and of small Stefan number. Using these asymptotic solutions, we found approximate expressions for the freezing, melting, and dissolution timescales, with freezing being the fastest effect and dissolution the slowest. In addition, using the earlytime solution we determined constraints on the parameters for which shell growth is possible. Our results predict that increasing the superheat or decreasing the particle size should decrease the time it takes for a particle to dissolve, in accordance with practical experience. Furthermore, the temperature perturbation is localised to a $5-10$ particle radii.

\subsection{Limitations and Outlook}

There are a number of assumptions that we have made that could be relaxed to extend the model. For example, we assumed that the density of the solid and molten cryolite are the same. We could investigate the effect of the density change when the cryolite freezes. We could also include advection in situations where the particles are larger, and we anticipate that this will break the radial symmetry that we have assumed in our analysis. Furthermore, we have neglected the effects of reaction-induced bubble generation, electromagnetic mixing, and turbulence in the bulk flow which all contribute to particle transport. Incorporating these effects into our model would make it more realistic. Finally, we could include additional particles, for example, by altering the far-field boundary conditions in the case when the particles are far apart. Addressing all these issues will form part of our future work.

The feeding and dissolution of alumina particles in the Hall-Héroult process involve several competing mechanisms which are challenging to couple in practical bathscale numerical simulations. The timescales illustrated in Figure 13 provide valuable input into the fineness of the timesteps required in these simulations if the particle-scale interactions are to be resolved. Furthermore, the models presented in this paper are useful as sub model or closure models in practical simulations if larger timesteps are used - as is often the case. The conditions under which no freezing occurs, highlighted in Figure 13, indicate the optimal regime in which the process should operate. Whether or not such conditions are possible to obtain in practice will be addressed in future work where our goal will be to combine the insight we have found for the single particle 
situation with state-of-the-art simulations and extensive industrial expertise to build a more realistic model for the feeding of alumina particles into the Hall-Héroult process.

\subsection{Acknowledgements}

This publication is based on work supported by the EPSRC Centre For Doctoral Training in Industrially Focused Mathematical Modelling (EP/L015803/1) in collaboration with Hydro Aluminium, NORCE and NTNU. Furthermore, this work is partly funded by SFI Metal Production, Centre for Research-based Innovation, 237738. Financial support from the Research Council of Norway and the partners of SFI Metal Production is gratefully acknowledged.

\section{References}

[1] K. Grjotheim, Aluminium Electrolysis. Fundamentals of the Hall-Heroult Process, Aluminium-Verlag, 1982.

[2] P. Lavoie, M. P. Taylor, J. B. Metson, A Review of Alumina Feeding and Dissolution Factors in Aluminum Reduction Cells, Metallurgical and Materials Transactions B 47 (2016) 26902696.

[3] D. I. Walker, Alumina in Aluminum Smelting and its behaviour after addition to cryolite-based electrolytes, Ph.D. thesis, University of Toronto, 1993

[4] Y. Yang, B. Gao, Z. Wang, Z. Shi, X. Hu, Study on the Dissolution of Alumina in Cryolite Electrolyte Using the See-Through Cell, Light Metals (2015).

[5] C. Kaszás, L. Kiss, S. Poncsák, S. Guérard, J.-F. Bilodeau, Spreading of Alumina and Raft Formation on the Surface of Cryolitic Bath, Light Metals (2017) 473-478.

[6] S. E. Gylver, N. H. Omdahl, A. K. Prytz, A. J. Meyer, L. P. Lossius, K. E. Einarsrud, Alumina feeding and raft formation: Raft collection and process parameters, in: C. Chesonis (Ed.), Light Metals 2019, Springer International Publishing, Cham, 2019, pp. 659-666.

[7] S. E. Gylver, A. Solheim, H. Gudbrandsen, Å. H. Follo, K. E. Einarsrud, Lab scale experiments on alumina raft formation, in: A. Tomsett (Ed.), Light Metals 2020, Springer International Publishing, Cham, 2020, pp. 659-663.

[8] J. Thonstad, F. Nordmo, J. B. Paulsen, Dissolution of alumina in molten cryolite, Metallurgical and Materials Transactions B 3 (1972) 407-412.

[9] J. Thonstad, A. Solheim, S. Rolseth, O. Skar, The Dissolution of Alumina in Cryolite Melts, Light Metals (1988) 105-111.

[10] R. Haverkamp, B. Welch, Modelling the dissolution of alumina powder in cryolite, Chemical Engineering and Processing: Process Intensification 37 (1998) 177-187.

[11] T. Hofer, Numerical Simulation and Optimization of the Alumina Distribution in an Aluminium Electrolysis Pot, Ph.D. thesis, École polytechnique fédérale de Lausanne, 2011.

[12] H. Zhang, S. Yang, H. Zhang, J. Li, Y. Xu, Numerical simulation of alumina-mixing process with a multicomponent flow model coupled with electromagnetic forces in aluminum reduction cells, Jom 66 (2014) 1210-1217.

[13] K. E. Einarsrud, I. Eick, W. Bai, Y. Feng, J. Hua, P. J. Witt, Towards a coupled multi-scale, multi-physics simulation framework for aluminium electrolysis, Applied Mathematical Modelling 44 (2017) 3-24.

[14] V. Bojarevics, Dynamic Modelling of Alumina Feeding in an Aluminium Electrolysis Cell, in: C. Chesonis (Ed.), Light Metals 2019, Springer International Publishing, Cham, 2019, pp. $675-682$.

[15] L. E. Scriven, On the dynamics of phase growth, Chemical Engineering Science 10 (1959) 1-13.
[16] J. L. Duda, J. S. Vrentas, Mathematical analysis of bubble dissolution, AIChE Journal 15 (1969) 351-356.

[17] J. L. Duda, J. S. Vrentas, Heat or mass transfer-controlled dissolution of an isolated sphere, International Journal of Heat and Mass Transfer 14 (1971) 395-407.

[18] R. I. Pedroso, G. A. Domoto, Perturbation Solutions for Spherical Solidification of Saturated Liquids, Journal of Heat Transfer 95 (1973) 42.

[19] D. S. Riley, F. T. Smith, G. Poots, The inward solidification of spheres and circular cylinders, International Journal of Heat and Mass Transfer 17 (1974) 1507-1516.

[20] K. Stewartson, R. T. Waechter, On Stefan's Problem for Spheres, Proceedings of the Royal Society A: Mathematical, Physical and Engineering Sciences 348 (1976) 415-426.

[21] M. A. Herrero, J. J. L. Velázquez, On the Melting of Ice Balls, SIAM Journal on Mathematical Analysis 28 (1997) 1-32.

[22] A. M. Soward, A Unified Approach to Stefan's Problem for Spheres and Cylinders, Proceedings of the Royal Society A: Mathematical, Physical and Engineering Sciences 373 (1980) $131-147$.

[23] S. W. McCue, J. R. King, D. S. Riley, Extinction behaviour for two-dimensional inward-solidification problems, Proceedings of the Royal Society A: Mathematical, Physical and Engineering Sciences 459 (2003) 977-999.

[24] S. W. McCue, B. Wu, J. M. Hill, Classical two-phase Stefan problem for spheres, Proceedings of the Royal Society A: Mathematical, Physical and Engineering Sciences 464 (2008) 2055-2076.

[25] J. N. Dewynne, J. M. Hill, Integral formulations and bounds for two phase Stefan problems initially not at their fusion temperature, Acta Mechanica 58 (1986) 201-228.

[26] H. E. Huppert, Phase changes following the initiation of a hot turbulent flow over a cold solid surface, Journal of Fluid Mechanics 198 (1989) 293.

[27] O. Ehrich, C. Yun-Ken, K. Schwerdtfeger, The melting of metal spheres involving the initially frozen shells with different material properties, International Journal of Heat and Mass Transfer 21 (1978) 341-349.

[28] D. W. Hahn, M. N. Ozisik, Heat conduction, John Wiley \& Sons, Inc., 2012.

[29] Hydro Aluminium, Internal Communications, 2019.

[30] AzoMaterials, Azo Materials, 2019.

[31] K. Grjotheim, H. Kvande, Introduction to Aluminium Electrolysis, 1993.

[32] A. Solheim, Aluminium Electrolysis Main Principles and Technology, 2009.

[33] P. N. Østbø, Evolution of Alpha Phase Alumina in Agglomerates upon Addition to Cryolitic Melts, Ph.D. thesis, Norwegian University of Science and Technology, 2002.

[34] S. W. Jessen, Mathematical Modeling of a Hall Héroult Aluminum Reduction Cell, $\{$ MSc. thesis $\}$, Technical University of Denmark, 2008.

[35] M. Kaviany, Principles of Heat Transfer in Porous Media, volume 3 of Mechanical Engineering Series, Springer New York, 1995.

[36] J. M. Back, Stefan problems for melting nanoscaled particles, Ph.D. thesis, Queensland University of Technology, 2016.

[37] F. Font, Beyond the classical Stefan problem, Ph.D. thesis, Universitat Politecnica de Catalunya, 2014. 